\title{
The first Dirichlet Eigenvalue of a Compact Manifold and the Yang Conjecture*
}

\author{
Jun LING
}

\begin{abstract}
We give a new estimate on the lower bound of the first Dirichlet eigenvalue of a compact Riemannian manifold with negative lower bound of Ricci curvature and provide a solution for a conjecture of H. C. Yang.
\end{abstract}

\section{Introduction}

It has been proved by $\mathrm{P}$. Li and $\mathrm{S}$. T. Yau 4 that if $M$ is an n-dimensional closed Riemannian manifold with Ricci curvature $\operatorname{Ric}(M)$ bounded below by $(n-1) \kappa$ with constant $\kappa<0$, then the first non-zero eigenvalue $\lambda$ of the Laplacian of $M$ has the lower bound

$$
\lambda \geq \frac{1}{2(n-1) d^{2}} \exp \left\{-1-\sqrt{1+4(n-1)^{2} d^{2}|\kappa|}\right\},
$$

where $d$ is the diameter of $M$. H. C. Yang $[8]$ improved the above estimate to the following

$$
\lambda \geq \frac{\pi^{2}}{d^{2}} \exp \left\{-C_{n} \sqrt{(n-1)|\kappa| d^{2}}\right\}
$$

where $C_{n}=\max \{\sqrt{n-1}, \sqrt{2}\}$. Yang further conjectured that

$$
\lambda \geq \frac{1}{2}(n-1) \kappa+\frac{\pi^{2}}{d^{2}} .
$$

If $M$ has a boundary, H. C. Yang conjectured that the above estimate holds for the first Dirichlet eigenvalue as well. In this paper, we give a new estimate on the lower bound of the first Dirichlet eigenvalue of an $n$-dimensional Riemannian manifold with negative lower bound of Ricci curvature. The result provides a solution for the conjecture of $\mathrm{H}$. C. Yang. Let $\operatorname{dist}(\cdot, \cdot)$ be the distance on $M$. We have the following result.

\footnotetext{
*2000 Mathematics Subject Classification Primary 58J50, 35P15; Secondary 53C21
} 
Theorem 1. If $(M, g)$ is an $n$-dimensional compact Riemannian manifold with boundary. Suppose that the boundary $\partial M$ of the manifold $M$ has nonnegative mean curvature with respect to the outward normal and that the Ricci curvature of $M$ has lower bound

$$
\operatorname{Ric}(M) \geq(n-1) \kappa
$$

for some constant $\kappa<0$. Then the first Dirichlet eigenvalue $\lambda$ of the Laplacian $\Delta$ of $M$ satisfies the inequality

$$
\lambda \geq \frac{1}{1-(n-1) \kappa /(2 \lambda)} \frac{\pi^{2}}{d^{2}}>0
$$

and $\lambda$ has the lower bound

$$
\lambda \geq \frac{1}{2}(n-1) \kappa+\frac{\pi^{2}}{d^{2}},
$$

where $d$ is the diameter of the largest interior ball in $M$,

$$
d=2 r \quad \text { and } \quad r=\max _{x \in M} \operatorname{dist}(x, \partial M) .
$$

If $\operatorname{Ric}(M) \geq(n-1) \kappa$ with constant $\kappa>0$, it is known that the first Dirichlet eigenvalue $\lambda$ has a lower bound as the above. Therefore the lower bound in (2) is universal for all three cases, constant $\kappa>0,=0$ or $<0$.

In the next section, we derive some preliminary estimates and conditions for test functions. In the last section we construct the needed test function and prove the main result. In the proof of the main result, instead of using the Zhong-Yang's canonical function or the "midrange" of the normalized eigenfunction of the first eigenvalue, we use a function $\xi$ that the author constructed in [5] to build the suitable test function.

\section{Preliminary Estimates}

Let $v$ be a normalized eigenfunction of the first Dirichlet eigenvalue of the Laplacian $\Delta$ such that

$$
\sup _{M} v=1, \quad \inf _{M} v=0 .
$$

The function $v$ satisfies the following

$$
\Delta v=-\lambda v \text { in } M
$$




$$
v=0 \quad \text { on } \partial M \text {. }
$$

We first use gradient estimate in [2]-4] and [7] to derive following estimate.

Lemma 1. The function $v$ satisfies the following

$$
\frac{|\nabla v|^{2}}{b^{2}-v^{2}} \leq \lambda(1+\beta)
$$

where $\beta=-(n-1) \kappa / \lambda>0$ and $b>1$ is an arbitrary constant.

Proof. Consider the function

$$
P(x)=|\nabla v|^{2}+A v^{2},
$$

where $A=\lambda-(n-1) \kappa+\epsilon$ for small $\epsilon>0$. The function $P$ must achieve its maximum at some point $x_{0} \in M$. We claim that

$$
\nabla P\left(x_{0}\right)=0 .
$$

If $x_{0} \in M \backslash \partial M$, (8) is obviously true. Suppose that $x_{0} \in \partial M$. Choose a local orthonormal frame $\left\{e_{1}, e_{2}, \cdots, e_{n}\right\}$ of $M$ about $x_{0}$ so that $e_{n}$ is the unit outward normal vector field near $x_{0} \in \partial M$ and $\left.\left\{e_{1}, e_{2}, \cdots, e_{n-1}\right\}\right|_{\partial M}$ is a local frame of $\partial M$ about $x_{0}$. The existence of such local frame can be justified as the following. Let $e_{n}$ be the local unit outward normal vector field of $\partial M$ about $x_{0} \in \partial M$ and $\left\{e_{1}, \cdots, e_{n-1}\right\}$ the local orthonormal frame of $\partial M$ about $x_{0}$. By parallel translation along the geodesic $\gamma(t)=\exp _{x_{0}} t e_{n}$, we may extend $e_{1}, \cdots, e_{n-1}$ to local vector fields of $M$. Then the extended frame $\left\{e_{1}, e_{2}, \cdots, e_{n}\right\}$ is what we need. Note that $\nabla_{e_{n}} e_{i}=0$ for $i \leq n-1$. Since $\left.v\right|_{\partial M}=0$, we have $v_{i}\left(x_{0}\right)=0$ for $i \leq n-1$.

$P\left(x_{0}\right)$ is the maximum implies that

$$
P_{i}\left(x_{0}\right)=0 \quad \text { for } i \leq n-1
$$

and

$$
P_{n}\left(x_{0}\right) \geq 0
$$


Using (3)-(5) in the following arguments, then we have that at $x_{0}$,

$$
\begin{aligned}
& \nabla_{e_{n}}\left(|\nabla v|^{2}\right)\left(x_{0}\right)=\sum_{i=1}^{n} 2 v_{i} v_{i n}=2 v_{n} v_{n n} \\
& =2 v_{n}\left(\Delta^{M} v-\sum_{i=1}^{n-1} v_{i i}\right)=2 v_{n}\left(-\lambda v-\sum_{i=1}^{n-1} v_{i i}\right) \\
& =-2 v_{n} \sum_{i=1}^{n-1} v_{i i}=-2 v_{n} \sum_{i=1}^{n-1}\left(e_{i} e_{i} v-\nabla_{e_{i}}^{M} e_{i} v\right) \\
& =2 v_{n} \sum_{i=1}^{n-1} \nabla_{e_{i}}^{M} e_{i} v=2 v_{n} \sum_{i=1}^{n-1} \sum_{j=1}^{n} g\left(\nabla_{e_{i}}^{M} e_{i}, e_{j}\right) v_{j} \\
& =2 v_{n}^{2} \sum_{i=1}^{n-1} g\left(\nabla_{e_{i}}^{M} e_{i}, e_{n}\right)=-2 v_{n}^{2} \sum_{i=1}^{n-1} g\left(\nabla_{e_{i}}^{M} e_{n}, e_{i}\right) \\
& =-2 v_{n}^{2} \sum_{i=1}^{n-1} h_{i i}=-2 v_{n}^{2}\left(x_{0}\right) m\left(x_{0}\right) \\
& \leq 0 \quad \text { by the non-negativity of } m,
\end{aligned}
$$

where $g($,$) is the Riemann metric of M,\left(h_{i j}\right)$ is the second fundamental form of $\partial M$ with respect to the outward normal $e_{n}$ and $m$ is the mean curvature of $\partial M$ with respect to $e_{n}$.

Noticing that $\left.v\right|_{\partial M}=0$, we have

$$
\left.P_{n}\left(x_{0}\right)=\left.\nabla_{e_{n}}\left(|\nabla v|^{2}\right)\right|_{x_{0}}+2 A v\left(x_{0}\right) v_{n}\left(x_{0}\right)\right) \leq 0 .
$$

Now (91), (10) and (12) imply that $P_{n}\left(x_{0}\right)=0$.

Thus (8) holds, no matter $x_{0} \notin \partial M$ or $x_{0} \in \partial M$. By (묘 and the Maximum Principle, we have

$$
\nabla P\left(x_{0}\right)=0 \quad \text { and } \quad \Delta P\left(x_{0}\right) \leq 0 .
$$

We are going to show further that $\nabla v\left(x_{0}\right)=0$. If on the contrary, $\nabla v\left(x_{0}\right) \neq 0$, then we rotate the local orthonormal frame about $x_{0}$ such that

$$
\left|v_{1}\left(x_{0}\right)\right|=\left|\nabla v\left(x_{0}\right)\right| \neq 0 \quad \text { and } \quad v_{i}\left(x_{0}\right)=0, \quad i \geq 2 .
$$

From (13) we have at $x_{0}$,

$$
0=\frac{1}{2} \nabla_{i} P=\sum_{j=1}^{n} v_{j} v_{j i}+A v v_{i},
$$




$$
v_{11}=-A v \quad \text { and } \quad v_{1 i}=0 \quad i \geq 2,
$$

and

$$
\begin{aligned}
0 & \geq \frac{1}{2} \Delta P\left(x_{0}\right)=\sum_{i, j=1}^{n}\left(v_{j i} v_{j i}+v_{j} v_{j i i}+A v_{i} v_{i}+A v v_{i i}\right) \\
& =\sum_{i, j=1}^{n}\left(v_{j i}^{2}+v_{j}\left(v_{i i}\right)_{j}+\mathrm{R}_{j i} v_{j} v_{i}+A v_{i i}^{2}+A v v_{i i}\right) \\
& =\sum_{i, j=1}^{n} v_{j i}^{2}+\nabla v \nabla(\Delta v)+\operatorname{Ric}(\nabla v, \nabla v)+A|\nabla v|^{2}+A v \Delta v \\
& \geq v_{11}^{2}+\nabla v \nabla(\Delta v)+(n-1) \kappa|\nabla v|^{2}+A|\nabla v|^{2}+A v \Delta v \\
& =(-A v)^{2}-\lambda|\nabla v|^{2}+(n-1) \kappa|\nabla v|^{2}+A|\nabla v|^{2}-\lambda A v^{2} \\
& =[A-\lambda+(n-1) \kappa]|\nabla v|^{2}+A(A-\lambda) v^{2}
\end{aligned}
$$

where we have used (14) and (11). Therefore at $x_{0}$,

$$
0 \geq[A-\lambda+(n-1) \kappa]|\nabla v|^{2}+A(A-\lambda) v^{2} .
$$

That is,

$$
\epsilon\left|\nabla v\left(x_{0}\right)\right|^{2}+[-(n-1) \kappa+\epsilon][\lambda-(n-1) \kappa+\epsilon] v\left(x_{0}\right)^{2} \leq 0 .
$$

Thus $\nabla v\left(x_{0}\right)=0$. This contradicts the assumption $\nabla v\left(x_{0}\right) \neq 0$.

Therefore we have $\nabla v\left(x_{0}\right)=0$, and

$$
P\left(x_{0}\right)=\left|\nabla v\left(x_{0}\right)\right|^{2}+A v\left(x_{0}\right)^{2}=A v\left(x_{0}\right)^{2} \leq A .
$$

Now for all $x \in M$ we have

$$
|\nabla v(x)|^{2}+A v(x)^{2}=P(x) \leq P\left(x_{0}\right) \leq A
$$

and

$$
|\nabla v(x)|^{2} \leq A\left(1-v(x)^{2}\right) .
$$

Letting $\epsilon \rightarrow 0$ in the above inequality, the estimate (6) follows. 
We want to improve the above upper bound in (6) further and proceed in the following way.

Define a function $Z$ on $\left[0, \sin ^{-1}(1 / b)\right]$ by

$$
Z(t)=\max _{x \in M, t=\sin ^{-1}(v(x) / b)} \frac{|\nabla v|^{2}}{b^{2}-v^{2}} / \lambda .
$$

The estimate in (6) becomes

$$
Z(t) \leq 1+\beta \quad \text { on }\left[0, \sin ^{-1}(1 / b)\right]
$$

Throughout this paper let

$$
\alpha=\frac{1}{2}(n-1) \kappa<0 \quad \text { and } \quad \delta=\alpha / \lambda<0 .
$$

We have the following conditions on the function $Z$.

Theorem 2. If the function $z:\left[0, \sin ^{-1}(1 / b)\right] \mapsto \mathbf{R}^{1}$ satisfies the following

1. $z(t) \geq Z(t) \quad t \in\left[0, \sin ^{-1}(1 / b)\right]$,

2. there exists some $x_{0} \in M$ such that at point $t_{0}=\sin ^{-1}\left(v\left(x_{0}\right) / b\right)$ $z\left(t_{0}\right)=Z\left(t_{0}\right)$,

3. $z\left(t_{0}\right) \geq 1$,

4. $z$ extends to a smooth even function, and

5. $z^{\prime}\left(t_{0}\right) \sin t_{0} \leq 0$

then we have the following

$$
0 \leq \frac{1}{2} z^{\prime \prime}\left(t_{0}\right) \cos ^{2} t_{0}-z^{\prime}\left(t_{0}\right) \cos t_{0} \sin t_{0}-z\left(t_{0}\right)+1-2 \delta \cos ^{2} t_{0} .
$$

Proof. Define

$$
J(x)=\left\{\frac{|\nabla v|^{2}}{b^{2}-v^{2}}-\lambda z\right\} \cos ^{2} t,
$$

where $t=\sin ^{-1}(v(x) / b)$. Then

$$
J(x) \leq 0 \quad \text { for } x \in M \quad \text { and } \quad J\left(x_{0}\right)=0 .
$$

So $J\left(x_{0}\right)$ is the maximum of $J$ on $M$. If $\nabla v\left(x_{0}\right)=0$, then

$$
0=J\left(x_{0}\right)=-\lambda z \cos ^{2} t .
$$


This contradicts the Condition 3 in the theorem. Therefore

$$
\nabla v\left(x_{0}\right) \neq 0
$$

We claim that

$$
\nabla J\left(x_{0}\right)=0
$$

If $x_{0} \in M \backslash \partial M$, (18) is obviously true. Suppose that $x_{0} \in \partial M$. Take the same local orthonormal frame $\left\{e_{1}, e_{2}, \cdots, e_{n}\right\}$ of $M$ about $x_{0}$ as in the proof of Lemma1, where $e_{n}$ is the unit outward normal vector field near $x_{0} \in \partial M$, $\left.\left\{e_{1}, e_{2}, \cdots, e_{n-1}\right\}\right|_{\partial M}$ is a local frame of $\partial M$ about $x_{0}$ and $\nabla_{e_{n}} e_{i}=0$ for $i \leq n-1$. Since $\left.v\right|_{\partial M}=0$, we have $v_{i}\left(x_{0}\right)=0$ for $i \leq n-1$. $J\left(x_{0}\right)$ is the maximum implies that

$$
J_{i}\left(x_{0}\right)=0 \quad \text { for } i \leq n-1
$$

and

$$
J_{n}\left(x_{0}\right) \geq 0 .
$$

Using argument in proving (11) and the non-negativity of the mean curvature $m$ of $\partial M$ with respect to the outward normal, we get

$$
\left.\left(|\nabla v|^{2}\right)_{n}\right|_{x_{0}} \leq 0
$$

The Dirichlet condition $v\left(x_{0}\right)=0$ implies that $t\left(x_{0}\right)=0$ and $z^{\prime}\left(t\left(x_{0}\right)\right)=$ $z^{\prime}(0)=0$, since by the Condition 4 in the theorem $z$ extends to a smooth even function. Therefore

$$
J_{n}\left(x_{0}\right)=\frac{1}{b^{2}}\left(|\nabla v|^{2}\right)_{n}-\left.\lambda \cos t\left[z^{\prime} \cos t-2 z \sin t\right] t_{n}\right|_{x_{0}}=\left.\frac{1}{b^{2}}\left(|\nabla v|^{2}\right)_{n}\right|_{x_{0}} \leq 0 .
$$

Now (19), (20) and (21) imply (18).

Thus (18) holds, no matter $x_{0} \notin \partial M$ or $x_{0} \in \partial M$. By (18) and the Maximum Principle, we have

$$
\nabla J\left(x_{0}\right)=0 \quad \text { and } \quad \Delta J\left(x_{0}\right) \leq 0 .
$$

$J(x)$ can be rewritten as

$$
J(x)=\frac{1}{b^{2}}|\nabla v|^{2}-\lambda z \cos ^{2} t
$$


Thus (22) is equivalent to

$$
\left.\frac{2}{b^{2}} \sum_{i} v_{i} v_{i j}\right|_{x_{0}}=\left.\lambda \cos t\left[z^{\prime} \cos t-2 z \sin t\right] t_{j}\right|_{x_{0}}
$$

and

$$
\begin{aligned}
0 \geq & \frac{2}{b^{2}} \sum_{i, j} v_{i j}^{2}+\frac{2}{b^{2}} \sum_{i, j} v_{i} v_{i j j}-\lambda\left(z^{\prime \prime}|\nabla t|^{2}+z^{\prime} \Delta t\right) \cos ^{2} t \\
& +4 \lambda z^{\prime} \cos t \sin t|\nabla t|^{2}-\left.\lambda z \Delta \cos ^{2} t\right|_{x_{0}} .
\end{aligned}
$$

Rotate the frame so that $v_{1}\left(x_{0}\right) \neq 0$ and $v_{i}\left(x_{0}\right)=0$ for $i \geq 2$. Then (23) implies

$$
\left.v_{11}\right|_{x_{0}}=\left.\frac{\lambda b}{2}\left(z^{\prime} \cos t-2 z \sin t\right)\right|_{x_{0}} \text { and }\left.\quad v_{1 i}\right|_{x_{0}}=0 \text { for } i \geq 2 .
$$

Now we have

$$
\begin{aligned}
\left.|\nabla v|^{2}\right|_{x_{0}} & =\left.\lambda b^{2} z \cos ^{2} t\right|_{x_{0}}, \\
\left.|\nabla t|^{2}\right|_{x_{0}} & =\frac{|\nabla v|^{2}}{b^{2}-v^{2}}=\left.\lambda z\right|_{x_{0}}, \\
\left.\frac{\Delta v}{b}\right|_{x_{0}} & =\Delta \sin t=\cos t \Delta t-\left.\sin t|\nabla t|^{2}\right|_{x_{0}}, \\
\left.\Delta t\right|_{x_{0}} & =\frac{1}{\cos t}\left(\sin t|\nabla t|^{2}+\frac{\Delta v}{b}\right) \\
\left.\Delta \cos ^{2} t\right|_{x_{0}} & =\left.\frac{1}{\cos t}\left[\lambda z \sin t-\frac{\lambda}{b} v\right]\right|_{x_{0}}, \quad \text { and } \\
& =-2 \lambda z \cos ^{2} t+\left.\frac{2}{b^{2}} \lambda v^{2}\right|_{x_{0}} .
\end{aligned}
$$

Therefore,

$$
\begin{aligned}
& \left.\frac{2}{b^{2}} \sum_{i, j} v_{i j}^{2}\right|_{x_{0}} \geq \frac{2}{b^{2}} v_{11}^{2} \\
& =\frac{\lambda^{2}}{2}\left(z^{\prime}\right)^{2} \cos ^{2} t-2 \lambda^{2} z z^{\prime} \cos t \sin t+\left.2 \lambda^{2} z^{2} \sin ^{2} t\right|_{x_{0}},
\end{aligned}
$$




$$
\begin{aligned}
&\left.\frac{2}{b^{2}} \sum_{i, j} v_{i} v_{i j j}\right|_{x_{0}}=\frac{2}{b^{2}}(\nabla v \nabla(\Delta v)+\operatorname{Ric}(\nabla v, \nabla v)) \\
& \geq \frac{2}{b^{2}}\left(\nabla v \nabla(\Delta v)+(n-1) \kappa|\nabla v|^{2}\right) \\
&=-2 \lambda^{2} z \cos ^{2} t+\left.4 \alpha \lambda z \cos ^{2} t\right|_{x_{0}} \\
&-\left.\lambda\left(z^{\prime \prime}|\nabla t|^{2}+z^{\prime} \Delta t\right) \cos ^{2} t\right|_{x_{0}} \\
&=-\lambda^{2} z z^{\prime \prime} \cos ^{2} t-\lambda^{2} z z^{\prime} \cos t \sin t \\
&+\left.\frac{1}{b} \lambda^{2} z^{\prime} v \cos t\right|_{x_{0}}
\end{aligned}
$$

and

$$
\begin{aligned}
& 4 \lambda z^{\prime} \cos t \sin t|\nabla t|^{2}-\left.\lambda z \Delta \cos ^{2} t\right|_{x_{0}} \\
& =4 \lambda^{2} z z^{\prime} \cos t \sin t+2 \lambda^{2} z^{2} \cos ^{2} t-\left.\frac{2}{b} \lambda^{2} z v \sin t\right|_{x_{0}} .
\end{aligned}
$$

Putting these results into (24) we get

$$
\begin{aligned}
0 \geq & -\lambda^{2} z z^{\prime \prime} \cos ^{2} t+\frac{\lambda^{2}}{2}\left(z^{\prime}\right)^{2} \cos ^{2} t+\lambda^{2} z^{\prime} \cos t(z \sin t+\sin t) \\
& +2 \lambda^{2} z^{2}-2 \lambda^{2} z+\left.4 \alpha \lambda z \cos ^{2} t\right|_{x_{0}},
\end{aligned}
$$

where we used (25). Now

$$
z\left(t_{0}\right)>0
$$

by Condition 3 in the theorem. Dividing two sides of (26) by $\left.2 \lambda^{2} z\right|_{x_{0}}$, we have

$$
\begin{aligned}
0 \geq & -\frac{1}{2} z^{\prime \prime}\left(t_{0}\right) \cos ^{2} t_{0}+\frac{1}{2} z^{\prime}\left(t_{0}\right) \cos t_{0}\left(\sin t_{0}+\frac{\sin t_{0}}{z\left(t_{0}\right)}\right)+z\left(t_{0}\right) \\
& -1+2 \delta \cos ^{2} t_{0}+\frac{1}{4 z\left(t_{0}\right)}\left(z^{\prime}\left(t_{0}\right)\right)^{2} \cos ^{2} t_{0} .
\end{aligned}
$$

Therefore,

$$
\begin{aligned}
0 \geq & -\frac{1}{2} z^{\prime \prime}\left(t_{0}\right) \cos ^{2} t_{0}+z^{\prime}\left(t_{0}\right) \cos t_{0} \sin t_{0}+z\left(t_{0}\right)-1+2 \delta \cos ^{2} t_{0} \\
& +\frac{1}{4 z\left(t_{0}\right)}\left(z^{\prime}\left(t_{0}\right)\right)^{2} \cos ^{2} t_{0}+\frac{1}{2} z^{\prime}\left(t_{0}\right) \sin t_{0} \cos t_{0}\left[\frac{1}{z\left(t_{0}\right)}-1\right]
\end{aligned}
$$


Conditions 2, 3 and 5 in the theorem imply that $z\left(t_{0}\right)=Z\left(t_{0}\right) \geq 1$ and $z^{\prime}\left(t_{0}\right) \sin t_{0} \leq 0$. Thus the last two terms in (28) are nonnegative and (17) follows.

\section{Proof of Theorem 1}

Proof of Theorem 1, Let

$$
z(t)=1+\delta \xi(t)
$$

where $\xi$ is the functions defined by (37) in Lemma 2, We claim that

$$
Z(t) \leq z(t) \quad \text { for } t \in\left[0, \sin ^{-1}(1 / b)\right] .
$$

Lemma 2 implies that for $t \in\left[0, \sin ^{-1}(1 / b)\right]$, we have the following

$$
\begin{aligned}
& \frac{1}{2} z^{\prime \prime} \cos ^{2} t-z^{\prime} \cos t \sin t-z=-1+2 \delta \cos ^{2} t, \\
& z^{\prime}(t) \sin t \leq 0, \quad(\text { since } \delta<0) \\
& z \text { is a smooth even function, and } \\
& z(t) \geq z\left(\frac{\pi}{2}\right)=1 .
\end{aligned}
$$

Let $P \in \mathbf{R}^{1}$ and $t_{0} \in\left[0, \sin ^{-1}(1 / b)\right]$ such that

$$
P=\max _{t \in\left[0, \sin ^{-1}(1 / b)\right]}(Z(t)-z(t))=Z\left(t_{0}\right)-z\left(t_{0}\right) .
$$

Thus

(35) $Z(t) \leq z(t)+P \quad$ for $t \in\left[0, \sin ^{-1}(1 / b)\right] \quad$ and $\quad Z\left(t_{0}\right)=z\left(t_{0}\right)+P$.

Suppose that $P>0$. Then $z+P$ satisfies the conditions in Theorem 2 and therefore satisfies (17). So we have

$$
\begin{aligned}
& z\left(t_{0}\right)+P=Z\left(t_{0}\right) \\
& \leq \frac{1}{2}(z+P)^{\prime \prime}\left(t_{0}\right) \cos ^{2} t_{0}-(z+P)^{\prime}\left(t_{0}\right) \cos t_{0} \sin t_{0}+1-2 \delta \cos ^{2} t_{0} \\
& =\frac{1}{2} z^{\prime \prime}\left(t_{0}\right) \cos ^{2} t_{0}-z^{\prime}\left(t_{0}\right) \cos t_{0} \sin t_{0}+1-2 \delta \cos ^{2} t_{0} \\
& =z\left(t_{0}\right) .
\end{aligned}
$$


This contradicts the assumption $P>0$. Thus $P \leq 0$ and (30) must hold. That along with the definition of the function $Z$ means

$$
\sqrt{\lambda} \geq \frac{|\nabla t|}{\sqrt{z(t)}}
$$

Take $q_{1}$ on $M$ such that $v\left(q_{1}\right)=1=\sup _{M} v$ and and $q_{2} \in \partial M$ such that distance $d\left(q_{1}, q_{2}\right)=$ distance $d\left(q_{1}, \partial M\right)$. Let $L$ be the minimum geodesic segment between $q_{1}$ and $q_{2}$. We integrate both sides of (36) along $L$ and change variable and let $b \rightarrow 1$. Let $d$ be, as in Theorem 1 the diameter of the largest interior ball in $M, d=2 r$ and $r=\max _{x \in M} \operatorname{dist}(x, \partial M)$. Then

$$
\begin{aligned}
\sqrt{\lambda} \frac{d}{2} & \geq \int_{L} \frac{|\nabla t(x)|}{\sqrt{z(t(x))}} d l=\int_{0}^{\frac{\pi}{2}} \frac{1}{\sqrt{z(t)}} d t \\
& \geq \frac{\left(\int_{0}^{\pi / 2} d t\right)^{\frac{3}{2}}}{\left(\int_{0}^{\pi / 2} z(t) d t\right)^{\frac{1}{2}}} \geq\left(\frac{\left(\frac{\pi}{2}\right)^{3}}{\int_{0}^{\pi / 2} z(t) d t}\right)^{\frac{1}{2}}
\end{aligned}
$$

Thus

$$
\lambda \geq \frac{\pi^{3}}{2 d^{2} \int_{0}^{\pi / 2} z(t) d t} .
$$

Now

$$
\int_{0}^{\frac{\pi}{2}} z(t) d t=\int_{0}^{\frac{\pi}{2}}[1+\delta \xi(t)] d t=\frac{\pi}{2}(1-\delta),
$$

by (40) in Lemma 2, Therefore we have

$$
\lambda \geq \frac{\pi^{2}}{(1-\delta) d^{2}} \quad \text { and } \quad \lambda \geq \frac{1}{2}(n-1) \kappa+\frac{\pi^{2}}{d^{2}} .
$$

We now present a lemma that is used in the proof of Theorem 1.

Lemma 2. Let

$$
\xi(t)=\frac{\cos ^{2} t+2 t \sin t \cos t+t^{2}-\frac{\pi^{2}}{4}}{\cos ^{2} t} \quad \text { on } \quad\left[-\frac{\pi}{2}, \frac{\pi}{2}\right] .
$$


Then the function $\xi$ satisfies the following

$$
\begin{aligned}
& \frac{1}{2} \xi^{\prime \prime} \cos ^{2} t-\xi^{\prime} \cos t \sin t-\xi=2 \cos ^{2} t \quad \text { in }\left(-\frac{\pi}{2}, \frac{\pi}{2}\right), \\
& \xi^{\prime} \cos t-2 \xi \sin t=4 t \cos t
\end{aligned}
$$$$
\int_{0}^{\frac{\pi}{2}} \xi(t) d t=-\frac{\pi}{2}
$$$$
1-\frac{\pi^{2}}{4}=\xi(0) \leq \xi(t) \leq \xi\left( \pm \frac{\pi}{2}\right)=0 \quad \text { on }\left[-\frac{\pi}{2}, \frac{\pi}{2}\right],
$$$$
\xi^{\prime} \text { is increasing on }\left[-\frac{\pi}{2}, \frac{\pi}{2}\right] \text { and } \xi^{\prime}\left( \pm \frac{\pi}{2}\right)= \pm \frac{2 \pi}{3} \text {, }
$$$$
\xi^{\prime}(t)<0 \text { on }\left(-\frac{\pi}{2}, 0\right) \text { and } \xi^{\prime}(t)>0 \text { on }\left(0, \frac{\pi}{2}\right),
$$$$
\xi^{\prime \prime}\left( \pm \frac{\pi}{2}\right)=2, \quad \xi^{\prime \prime}(0)=2\left(3-\frac{\pi^{2}}{4}\right) \text { and } \xi^{\prime \prime}(t)>0 \text { on }\left[-\frac{\pi}{2}, \frac{\pi}{2}\right],
$$$$
\left(\frac{\xi^{\prime}(t)}{t}\right)^{\prime}>0 \text { on }(0, \pi / 2) \text { and } 2\left(3-\frac{\pi^{2}}{4}\right) \leq \frac{\xi^{\prime}(t)}{t} \leq \frac{4}{3} \text { on }\left[-\frac{\pi}{2}, \frac{\pi}{2}\right] \text {, }
$$$$
\xi^{\prime \prime \prime}\left(\frac{\pi}{2}\right)=\frac{8 \pi}{15}, \xi^{\prime \prime \prime}(t)<0 \text { on }\left(-\frac{\pi}{2}, 0\right) \text { and } \xi^{\prime \prime \prime}(t)>0 \text { on }\left(0, \frac{\pi}{2}\right) .
$$

Proof. For convenience, let $q(t)=\xi^{\prime}(t)$, i.e.,

$$
q(t)=\xi^{\prime}(t)=\frac{2\left(2 t \cos t+t^{2} \sin t+\cos ^{2} t \sin t-\frac{\pi^{2}}{4} \sin t\right)}{\cos ^{3} t} .
$$

Equation (38) and the values $\xi\left( \pm \frac{\pi}{2}\right)=0, \xi(0)=1-\frac{\pi^{2}}{4}$ and $\xi^{\prime}\left( \pm \frac{\pi}{2}\right)= \pm \frac{2 \pi}{3}$ can be verified directly from (37) and (41). The values of $\xi^{\prime \prime}$ at 0 and $\pm \frac{\pi}{2}$ can be computed via (38). By (39), $\left(\xi(t) \cos ^{2} t\right)^{\prime}=4 t \cos ^{2} t$. Therefore $\xi(t) \cos ^{2} t=\int_{\frac{\pi}{2}}^{t} 4 s \cos ^{2} s d s$, and

$$
\begin{aligned}
& \int_{-\frac{\pi}{2}}^{\frac{\pi}{2}} \xi(t) d t=2 \int_{0}^{\frac{\pi}{2}} \xi(t) d t=-8 \int_{0}^{\frac{\pi}{2}}\left(\frac{1}{\cos ^{2}(t)} \int_{t}^{\frac{\pi}{2}} s \cos ^{2} s d s\right) d t \\
= & -8 \int_{0}^{\frac{\pi}{2}}\left(\int_{0}^{s} \frac{1}{\cos ^{2}(t)} d t\right) s \cos ^{2} s d s=-8 \int_{0}^{\frac{\pi}{2}} s \cos s \sin s d s=-\pi .
\end{aligned}
$$

It is easy to see that $q$ and $q^{\prime}$ satisfy the following equations

$$
\frac{1}{2} q^{\prime \prime} \cos t-2 q^{\prime} \sin t-2 q \cos t=-4 \sin t,
$$


and

$$
\frac{\cos ^{2} t}{2\left(1+\cos ^{2} t\right)}\left(q^{\prime}\right)^{\prime \prime}-\frac{2 \cos t \sin t}{1+\cos ^{2} t}\left(q^{\prime}\right)^{\prime}-2\left(q^{\prime}\right)=-\frac{4}{1+\cos ^{2} t} .
$$

The last equation implies $q^{\prime}=\xi^{\prime \prime}$ cannot achieve its non-positive local minimum at a point in $\left(-\frac{\pi}{2}, \frac{\pi}{2}\right)$. On the other hand, $\xi^{\prime \prime}\left( \pm \frac{\pi}{2}\right)=2$, by equation (38), $\xi\left( \pm \frac{\pi}{2}\right)=0$ and $\xi^{\prime}\left( \pm \frac{\pi}{2}\right)= \pm \frac{2 \pi}{3}$. Therefore $\xi^{\prime \prime}(t)>0$ on $\left[-\frac{\pi}{2}, \frac{\pi}{2}\right]$ and $\xi^{\prime}$ is increasing. Since $\xi^{\prime}(t)=0$, we have $\xi^{\prime}(t)<0$ on $\left(-\frac{\pi}{2}, 0\right)$ and $\xi^{\prime}(t)>0$ on $\left(0, \frac{\pi}{2}\right)$. Similarly, from the equation

$$
\begin{gathered}
\frac{\cos ^{2} t}{2\left(1+\cos ^{2} t\right)}\left(q^{\prime \prime}\right)^{\prime \prime}-\frac{\cos t \sin t\left(3+2 \cos ^{2} t\right)}{\left(1+\cos ^{2} t\right)^{2}}\left(q^{\prime \prime}\right)^{\prime}-\frac{2\left(5 \cos ^{2} t+\cos ^{4} t\right)}{\left(1+\cos ^{2} t\right)^{2}}\left(q^{\prime \prime}\right) \\
=-\frac{8 \cos t \sin t}{\left(1+\cos ^{2} t\right)^{2}}
\end{gathered}
$$

we get the results in the last line of the lemma.

Set $h(t)=\xi^{\prime \prime}(t) t-\xi^{\prime}(t)$. Then $h(0)=0$ and $h^{\prime}(t)=\xi^{\prime \prime \prime}(t) t>0$ in $\left(0, \frac{\pi}{2}\right)$. Therefore $\left(\frac{\xi^{\prime}(t)}{t}\right)^{\prime}=\frac{h(t)}{t^{2}}>0$ in $\left(0, \frac{\pi}{2}\right)$. Note that $\frac{\xi^{\prime}(-t)}{-t}=\frac{\xi^{\prime}(t)}{t}$, $\left.\frac{\xi^{\prime}(t)}{t}\right|_{t=0}=\xi^{\prime \prime}(0)=2\left(3-\frac{\pi^{2}}{4}\right)$ and $\left.\frac{\xi^{\prime}(t)}{t}\right|_{t=\pi / 2}=\frac{4}{3}$. This completes the proof of the lemma.

\section{References}

[1] I. Chavel. Eigenvalues in Riemannian Geometry, Academic Press, Orlando, Fla, 1984.

[2] P. Li. Lecture Notes on Geometric Analysis, Lecture Notes Series, \#6, Seoul National University, Seoul, Korea.

[3] P. Li and S. T. Yau. On the Schrödinger equation and the eigenvalue problem, Comm. Math. Phys., 88(1983), 309-318.

[4] P. Li and S. T. Yau. Estimates of eigenvalues of a compact Riemannian manifold, AMS Proc. Symp. Pure Math., 36(1980), 205-239.

[5] Jun Ling. A Bound for the First Fundamental Gap. Ph.D. Dissertation, State University of New York at Buffalo.

[6] Jun Ling. Estimates on the lower bound of the first gap, Preprint, 2004.

[7] R. Schoen, and S. T. Yau. Lecture Notes on Differential Geometry, Conference Procedings and Lecture Notes in Geometry and Topology, Vol 1, International Press, 1994. 
[8] H. C. Yang. Estimate for the first eigenvalue for a compact Riemannian manifold, Science in China, Ser. A 33,(1990), 39-51.

[9] S. T. Yau, editor. Problem Section, Seminar on Diff. Geom., Princeton University Press, Princeton, 1982.

[10] J.-Q. Zhong and H. C. Yang. On the estimate of the first eigenvalue of a compact Riemannian manifod, Sci. Sinica, Ser. A 27, (1984), $1265-1273$.

Department of mathematics, Utah Valley State College, Orem, Utah 84058

E-mail address: lingju@uvsc.edu 